\title{
Evaluation of Interleukin-23 Receptor Gene Polymorphism in Vitiligo Patients F.M.Elesawy ${ }^{1}$, N.F.Ibrahim ${ }^{2}$, A.I.Mustafa ${ }^{1}$ and S.H.Aly ${ }^{1}$ ${ }^{1}$ Dermatology,Venereology,Andrology Dept., Faculty of Medicine, Benha Univ., Benha, Egypt ${ }^{2}$ Biochemistry, Molecular Biology Dept.,Faculty of Medicine, Benha Univ., Benha, Egypt
}

\section{Abstract}

E-Mail:samah@gmail.com

Vitiligo is a common, acquired pigmentary disorder of unknown etiology, affecting up to $0.1-2 \%$ of the population worldwide. Interleukin-23 (IL-23) is a cytokine which is believed to have an important role in the pathogenesis of autoimmune diseases. The aim of our study was to investigate the association between IL-23 (p2199A/C) receptor gene polymorphism and vitiligo pathogenesis. This was a case control study which included 50 patients suffering from vitiligo (Group A). In addition to 50 apparently healthy individuals of matched age and sex had been chosen as a control group (Group B). Each patient was subjected to full history taking, complete clinical examination, and Laboratory investigations for serum level of Il23 receptor gene. The mean age of studied cases was 30.8 years. They were 16 males (32\%) and 34 females $(68 \%)$. In addition, 50 apparently healthy individuals of matched age and sex was added as a control group ( $\mathrm{P}>0.05)$. Taking rs10889677 CC as the reference genotype and $\mathrm{C}$ as the reference allele; AA genotype and A allele showed significantly higher frequency when compared to control groups ( $\mathrm{p}=0.024,0.049$ respectively), with risk to develop vitiligo. BSA\%, disease duration, frequency of progressive disease increased gradually, while age of disease onset decreased gradually according to CC, CA, and AA respectively. The rs10889677 AA genotype and A allele showed significantly higher frequency in cases when compared to control groups with risk to develop vitiligo cases.

Keywords: Interleukin-23, Gene, Polymorphism,Vitiligo.

\section{Introduction}

Vitiligo is an acquired depigmentation disorder, manifests as white macules on the skin from which melanocytes (pigment-producing cells) have been lost and can cause significant psychological stress and stigmatization [1].

Vitiligo affects about $1 \%$ of people worldwide. The most common type is non-segmental generalized vitiligo (hereafter referred to as vitiligo), which presents with widely distributed, usually symmetric, and progressive lesions. It has a pronounced impact on the physical and mental health of patients, including loss of skin photoprotection, compromised cutaneous immunity, and an appreciable reduction in quality of life that is directly correlated with the early age of onset (typically in the first two decades of life) [2].

Diverse mechanisms that may contribute to the etiology of vitiligo have been proposed; these include autoimmune, genetic, biochemical, oxidative stress, neural, and viral factors. Vitiligo has also been associated with other autoimmune diseases, for example, autoimmune thyroid disease, rheumatoid arthritis, diabetes mellitus, psoriasis, and lupus erythematosus, among others [3].

Many studies have been made to reveal the pathophysiology of vitiligo. A trigger event which range from sunburn to mechanical trauma and chemical exposures, is thought to instigate stress responses in the skin that elicit an autoimmune response in genetically susceptible individuals that ultimately targets the melanocytes known to have an inherited fragility, predisposing individuals to develop vitiligo [4].

The most significant progress in our understanding of disease etiology has been made on three fronts: characterizing the stress responses activated by vitiligo triggers, delineating the autoimmune components that cause disease progression, and identifying susceptibility genes [5].

The IL-23R gene is located on chromosome 1p31. It is highly expressed in dendritic cells, and is involved in several chronic inflammatory diseases. The interleukin23 receptor (IL-23R) is composed of IL-23R and IL$12 \mathrm{R} \beta 1$ subunits, which is shared with IL-12R [6].

IL-23/IL-23R is essential for the T-helper 17 (Th17) cell-mediated immune response.

IL-23R plays an important role in the initiating, maintaining and accelerating the IL-23/IL-17 inflammatory signal transduction pathway [7].

Since then, IL23Rgene was proved to be the predisposing gene to many other autoimmune/inflammatory diseases. Certain SNP alleles in the IL-23R gene can up-regulate its expression on several immune cells, and amplify the inflammatory condition [8].

The aim of the study was to investigate the association between IL-23 (b2199A/C) receptor gene polymorphism and vitiligo pathogenesis.

\section{Patients and methods}

This case-control study included 50 patients suffering from vitiligo (Group A). In addition to 50 apparently healthy individuals of matched age and sex had been chosen as a control group (Group B). All patients were selected from the outpatient clinic of Dermatology and Andrology Department of Benha University Hospitals in the period between March and May 2019.

The study was approved by the local ethics committee on research involving human subjects of Benha Faculty of Medicine.An Informed consent was obtained from each individual before sample collection.

Each patient was subjected to full history taking, complete clinical examination, and Laboratory investigations for serum level of Il23 receptor gene. 


\subsection{Detection of $\mathbf{I L 2 3}$ receptor gene polymorphism by PCR}

PCR is a test for in vitro amplification of specific DNA sequences using two primers that hybridize to opposite strands and flank target DNA region.

Sequences flanking IL23 I/D polymorphism were PCR-amplified from genomic DNA using a pair of specific oligonucleotide primers, The amplified products were then analyzed by electrophoresis. The insertion allele (I) was detected as a $490 \mathrm{bp}$ band and the delection (D) allele was visualized as $190 \mathrm{bp}$ band. Both bands were detected in I/D genotype.

\subsection{Statistical analysis}

Data were statistically described in terms of mean \pm standard deviation (Mean $\pm \mathrm{SD}$ ), median and range, or frequencies (number of cases) and percentages when appropriate. Comparison of numerical variables between the study groups was done using Student $t$ test (t-test) for independent samples in comparing 2 groups when normally distributed. The test statistics used to measure the statistical relationship, or association, between two continuous variables is Pearson's correlation coefficient (r-test). The non-parametric test (Spearman's Rho-test) was used to measure the strength of association between two variables. Fisher's exact test (F-test) was used to know whether the proportions for one variable are different among values of the other variable. Exact test was used instead when the expected frequency is less than 5. P-values less than 0.05 was considered statistically significant. All statistical calculations were tabulated and analyzed using computer programs SPSS (Statistical Package for the Social Science; SPSS Inc., Chicago, IL, USA) version 16 for Microsoft Windows.

\section{Results}

This study included 50 patients suffering from vitiligo. The mean age of studied cases was 30.8 years. They were 26 males (42\%) and 24 females (58\%). In addition, 50 apparently healthy individuals of matched age and sex was added as a control group.

The rs10889677 SNP had A and C alleles. C is the reference. It is located on the short (p) arm of chromosome 1 at position 31.3, within IL23R gene.

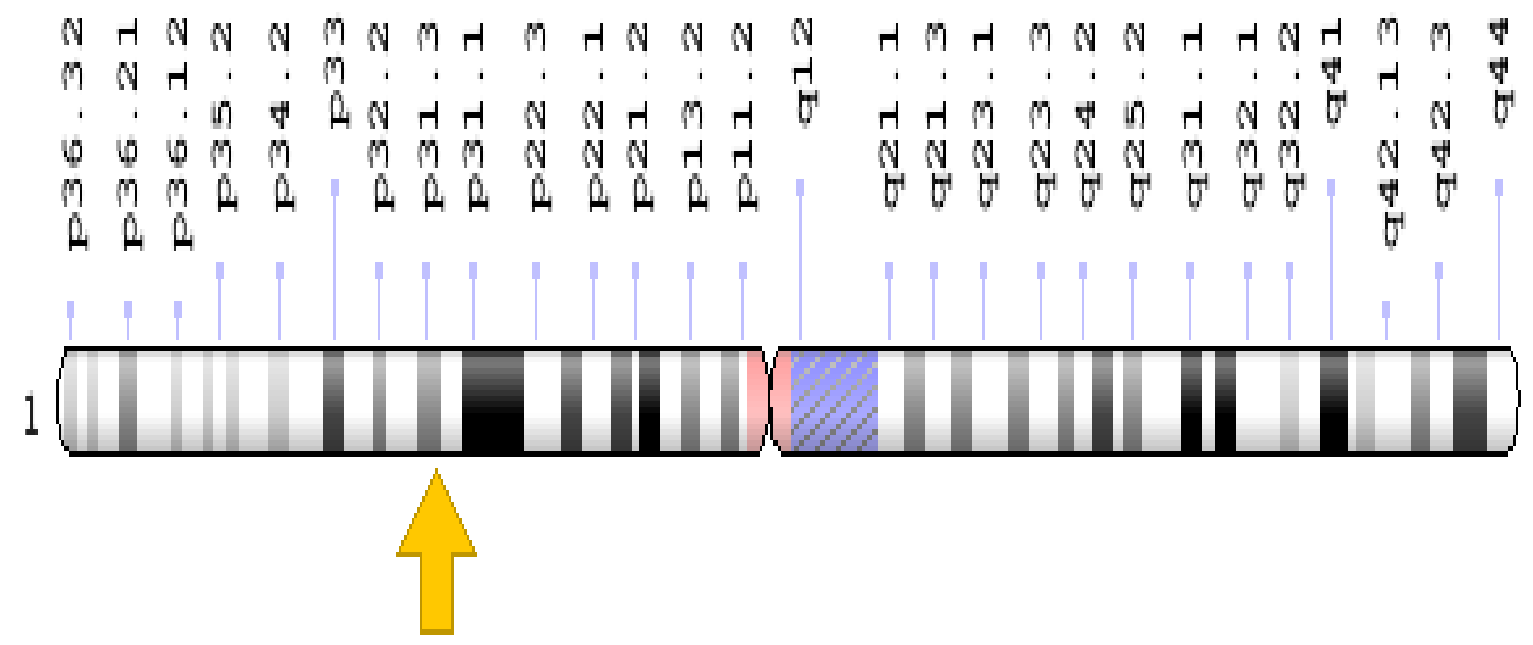

Fig (1) Chromosome 1, arrow indicates 1p31.3.

The cases and control subjects were selected randomly from Qaliobeya Governorate in Egypt. They were unrelated. Hardy Weinberg equation was applied. It revealed that rs10889677 genotypes in control as well as in cases, groups were in equilibrium.

Taking rs10889677 CC as the reference genotype and $\mathrm{C}$ as the reference allele; AA genotype and A allele showed significantly higher frequency when compared to control groups $(\mathrm{p}=0.024,0.049$ respectively), with risk to develop vitiligo $(\mathrm{OR}=3.844,95 \% \mathrm{CI}=1.190-12.420$; $\mathrm{OR}=1.839$, $\mathrm{OR}=1.002-3.372$ respectively) as shown in Fig (2).
BSA \%, disease duration, frequency of progressive disease increased gradually, while age of disease onset decreased gradually according to $\mathrm{CC}, \mathrm{CA}$, and AA respectively. Site of affection and clinical types did not differ significantly between IL23R genotypes in all studied cases, Table (2).

Logistic regression analysis was conducted for prediction of vitiligo susceptibility using age, gender, FH, smoking, rs10889677 genotypes as covariates. Family history, smoking and AA genotype were considered risk predictors for vitiligo susceptibility in uni- and multivariable analyses. 


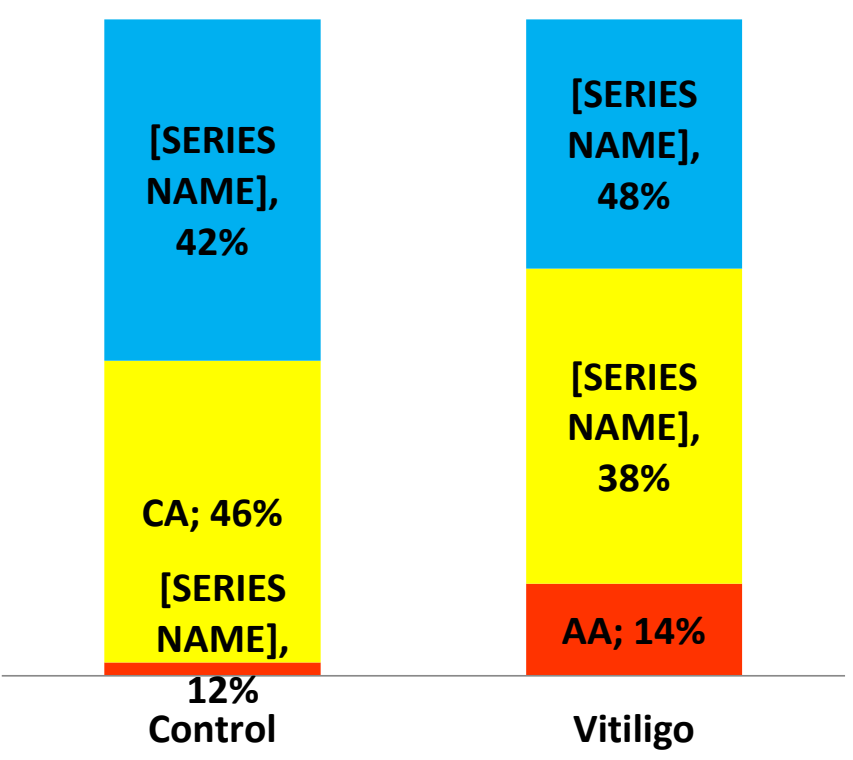

Fig (2) IL23R rs10889677 genotypes in control and cases groups.

Table (2) Comparison of genotype distribution rs10889677 regarding clinical data in all studied vitiligo cases.

\begin{tabular}{|c|c|c|c|c|c|c|c|c|c|}
\hline \multirow{2}{*}{\multicolumn{2}{|c|}{ Body surface area(BSA) }} & \multirow[b]{2}{*}{ mean \pm SD } & \multicolumn{2}{|c|}{$\begin{array}{c}\mathrm{CC} \\
\mathrm{N}=19\end{array}$} & \multicolumn{2}{|c|}{$\begin{array}{c}\mathrm{CA} \\
\mathrm{N}=24\end{array}$} & \multicolumn{2}{|c|}{$\begin{array}{c}\text { AA } \\
\mathrm{N}=7\end{array}$} & \multirow{2}{*}{$\begin{array}{c}\boldsymbol{P} \\
<0.001\end{array}$} \\
\hline & & & 5.3 & 1.7 & 38.5 & 11.5 & 51.6 & 16.2 & \\
\hline \multicolumn{2}{|c|}{ Age of Onset (years) } & mean $\pm \mathrm{SD}$ & 39.6 & 11.1 & 14.6 & 4.8 & 9.1 & 2.6 & $<0.001$ \\
\hline \multicolumn{2}{|c|}{ Duration (years) } & mean $\pm \mathrm{SD}$ & 2.8 & 0.9 & 8.9 & 2.1 & 17.6 & 5.2 & $<0.001$ \\
\hline \multirow[t]{2}{*}{ Course } & Stationary & $\mathrm{N}, \%$ & 8 & $42.1 \%$ & 4 & $16.7 \%$ & 0 & $0 \%$ & \multirow{2}{*}{0.045} \\
\hline & Progressive & $\mathrm{N}, \%$ & 11 & $57.9 \%$ & 20 & $83.3 \%$ & 4 & $100 \%$ & \\
\hline \multirow[t]{3}{*}{ Site } & Head and neck & $\mathrm{N}, \%$ & 10 & $53.2 \%$ & 17 & $70.8 \%$ & 8 & $57.1 \%$ & 0.774 \\
\hline & Extremities & $\mathrm{N}, \%$ & 17 & $89.5 \%$ & 21 & $87.5 \%$ & 6 & $100 \%$ & 0.622 \\
\hline & Trunk & $\mathrm{N}, \%$ & 8 & $41.6 \%$ & 8 & $33.3 \%$ & 1 & $0 \%$ & 0.264 \\
\hline \multirow[t]{3}{*}{ Type } & Vulgaris & $\mathrm{N}, \%$ & 6 & $41.6 \%$ & 10 & $41.7 \%$ & 2 & $28.6 \%$ & 0.717 \\
\hline & Focal & $\mathrm{N}, \%$ & 11 & $47.9 \%$ & 14 & $58.3 \%$ & 4 & $57.1 \%$ & 0.998 \\
\hline & Acrofacial & $\mathrm{N}, \%$ & 2 & $10.5 \%$ & 0 & $0 \%$ & 1 & $14.3 \%$ & 0.161 \\
\hline
\end{tabular}

\section{Discussion}

Interleukin-23 (IL-23) is a cytokine which is believed to have an important role in the pathogenesis of autoimmune diseases. Therefore, it can be used as a target molecule in the treatment of autoimmune disease, the production of IL-17A and IL-17F by Tlymphocytesis regulated proximally by IL-23 produced by dendriticcells, independently of cell-to-cell contact or traditional TCR activation. IL-23 is a member of the IL12 family, which activates the effector function of Th17 cells to pro-mote inflammatory responses. It has been established thatIL-23 is essential for the development of autoimmune diseases, including psoriatic skin inflammation, inflammatorybowel disease, autoimmune diabetes, and experimental autoimmune encephalomyelitis. This cytokine induces theproduction of Th17 cells, so it was thought that IL-23mediates autoimmunity by secretion of the IL-17 family.8In patients with vitiligo, increased serum IL-17 levels were observed, suggesting that IL-17 may participate in the immune response in early-onset disease [8].
IL-23 polymorphism was linked to many autoimmune diseases like autoimmune uveitis [10], psoriasis [11], alopecia areata [12], and IBD [13].

In analyzing Comparison of genotypes distribution rs10889677 in all studied vitiligo cases, No significant differences were found in demographic data, risk factors, Site of affection and clinical types, between rs 10889677 genotypes in all studied vitiligo patients.

Otherwise, BSA\%, disease duration, frequency of progressive disease increased gradually, while age of disease onset decreased gradually according to $\mathrm{CC}, \mathrm{CA}$, AA respectively.

\section{Conclusion}

The rs10889677 AA genotype and A allele showed significantly higher frequency in cases when compared to control groups with risk to develop vitiligo cases. VASI score, disease duration, frequency of progressive disease increased gradually, while age of disease onset decreased gradually according to $\mathrm{CC}, \mathrm{CA}, \mathrm{AA}$ respectively. Family history, smoking and AA genotype were considered risk predictors for vitiligo susceptibility. 
Younger age of onset, longer disease duration, CA and AA genotypes were considered independent predictors of more severe vitiligo cases.

\section{References}

[1] P. R. Mangan . "Transforming growth factor- $\beta$ induces development of the TH 17 lineage," Nature, Vol.441, PP.231-234, 2006.

[2] D. Parsad, S. Dogra, A. J. Kanwar, "Quality of life in patients with vitiligo," Health Qual. Life Outcomes, Vol.1, PP.1-3, 2003.

[3] Y. Zhang ."The prevalence of vitiligo: a metaanalysis," PLoS One, Vol.11, p. e0163806, 2016.

[4] R. E. Boissy and P. Manga, "On the etiology of contact/occupational vitiligo. Pigment Cell Res., Vol.17, PP.208-214, 2004.

[5] K. Ezzedine, V. Eleftheriadou, M. Whitton, and N. van Geel, "Vitiligo," Lancet, Vol.386, PP.74-84, 2015.

[6] C. Parham . "A receptor for the heterodimeric cytokine IL-23 is composed of IL-12R $\beta 1$ and a novel cytokine receptor subunit, IL-23R," J. Immunol., Vol.168, PP.5699-5708, 2002.

[7] M. Zhang ."Functional polymorphisms in interleukin23 receptor and susceptibility to coronary artery disease," DNA Cell Biol., Vol.33, PP.891-897, 2014.
[8] E. Duvallet, L. Semerano, E. Assier, G. Falgarone, M.-C. Boissier, "Interleukin-23: a key cytokine in inflammatory diseases," Ann. Med., Vol.43, PP.503$511,2011$.

[9] A. Di Cesare, P. Di Meglio, and F. O. Nestle, "The IL-23/Th17 axis in the immunopathogenesis of psoriasis," J. Invest. Dermatol., Vol.129, PP.13391350, 2009.

[10] Z. Zhong, G. Su, A. Kijlstra, and P. Yang, "Activation of the interleukin-23/interleukin-17 signalling pathway in autoinflammatory and autoimmune uveitis," Prog. Retin. Eye Res., Vol.2020, p. 100866, 2020.

[11] J. E. Hawkes, B. Y. Yan, T. C. Chan, J. G. Krueger, "Discovery of the IL-23/IL-17 signaling pathway and the treatment of psoriasis," J. Immunol., Vol.201, PP.1605-1613, 2018.

[12] L. S. Ortolan . "IL-12/IL-23 neutralization is ineffective for alopecia areata in mice and humans," J. Allergy Clin. Immunol., Vol.144, PP.1731-1734, 2019.

[13] A. R. Moschen, H. Tilg, and T. Raine, "IL-12, IL-23 and IL-17 in IBD: immunobiology and therapeutic targeting," Nat. Rev. Gastroenterol. Hepatol., Vol.16, PP.185-196, 2019. 